\title{
INVESTIGATION OF DATA FUSION APPLIED TO HEALTH MONITORING OF WIND TURBINE DRIVETRAIN COMPONENTS
}

\author{
Paula J. Dempsey \\ NASA Glenn Research Center \\ Cleveland, Ohio 44135 \\ Paula.J.Dempsey@nasa.gov \\ Shuangwen Sheng \\ National Renewable Energy Laboratory \\ Golden, Colorado 80401 \\ Shuangwen.Sheng@nrel.gov
}




\section{TABLE OF CONTENTS}

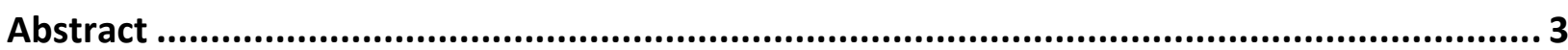

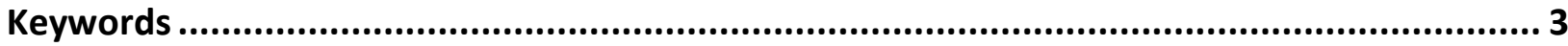

Introduction ....................................................................................................... 4

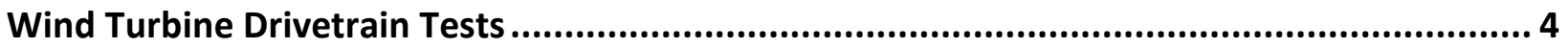

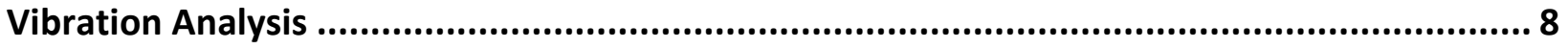

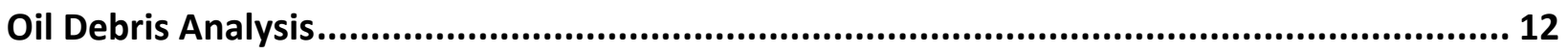

Data Fusion .................................................................................................... 13

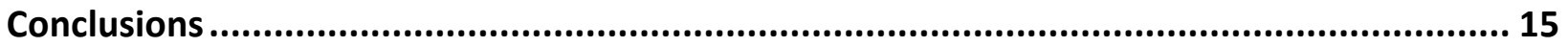

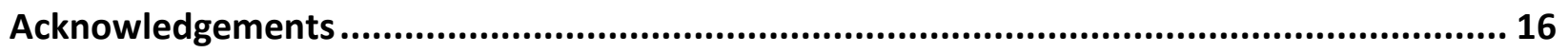

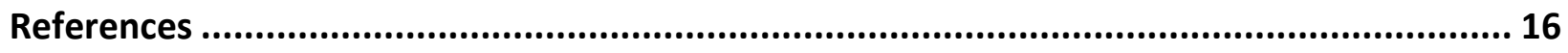




\section{ABSTRACT}

The research described was performed on diagnostic tools used to detect damage to dynamic mechanical components in a wind turbine gearbox. Different monitoring technologies were evaluated by collecting vibration and oil debris data from tests performed on a "healthy" gearbox and a damaged gearbox in a dynamometer test stand located at the National Renewable Energy Laboratory. The damaged gearbox tested was removed from the field after experiencing component damage due to two losses of oil events and was retested under controlled conditions in the dynamometer test stand. Preliminary results indicate oil debris and vibration can be integrated to assess the health of the wind turbine gearbox.

\section{KEYWORDS}

Data fusion, health monitoring, oil debris analysis, vibration analysis, wind turbine drivetrain 


\section{INTRODUCTION}

The development of an online condition-monitoring system for detecting damage to wind turbine dynamic mechanical components is essential for improved safety and reduced operational costs [1]. A condition-monitoring system will help achieve predictive, condition-based maintenance that improves safety, decreases maintenance costs, and increases system availability [2]. Incorporating data fusion methods into a condition-monitoring system to detect early onset of fault conditions and their progression would enable wind turbine maintenance actions to be planned. Studies have shown that data fusion methods-integrating several different diagnostic tools - result in a diagnostic system with improved detection and reduced false alarms, as compared to individual diagnostic tools [3, 4]. Automated data fusion processes can aid decision making by refining and reducing the quantity of information the wind turbine operators must examine, enabling a timely, robust, and relevant assessment of the situation.

The objective of this research is to demonstrate vibration-based health-monitoring techniques, oil debris analysis techniques, and wind turbine operational parameters combined to provide improved detection and decision-making capabilities as compared to individual diagnostic tools. This hypothesis is evaluated using data collected from two wind turbine gearboxes tested at the National Renewable Energy Laboratory (NREL) in a dynamometer test stand. The gearboxes used were identical except for manufacturing variances. Vibration and oil debris data were collected along with torque and speed data. Baseline data was collected on the dynamometer test stand from a test gearbox with "healthy" drivetrain components. Data then was collected from an identical gearbox after its internal components had sustained damage. Some damage to the wind turbine gear sets located on the high-speed shaft and the intermediate-speed shaft occurred in the field; the gearbox was removed and retested under controlled conditions in the test stand. The damage in the field mainly was caused by oil loss in the gearbox occurring on two separate occasions. Details of the test stand and analysis of the vibration and oil debris data are discussed.

\section{WIND TURBINE DRIVETRAIN TESTS}

The wind turbine drivetrain tests were performed in the dynamometer test facility located at NREL. The test facility was developed to conduct performance and reliability tests on wind turbine drivetrain prototypes and commercial machines $[5,6]$. The facility is capable of providing static, highly accelerated life, and model-in-the-loop tests. The prime mover of the dynamometer is composed of a 2.5-MW induction motor, a 3-stage epicyclical reducer, and a variable-frequency drive with full regeneration capacity. The rated torque provided by the dynamometer to a test article can be up to 1.4 meganewton meter with speed varying from $0 \mathrm{rpm}$ to $16.7 \mathrm{rpm}$. Non-torque loading actuators, rated up to $440 \mathrm{kN}$ for radial and $156 \mathrm{kN}$ for thrust load, also can be utilized in the dynamometer to apply great thrust, bending, and shear loads normally generated by a wind turbine's rotor. Figure 1(a) is a diagram of the test facility and Figure 1(b) is a photo of the test implementation with the test article installed. 


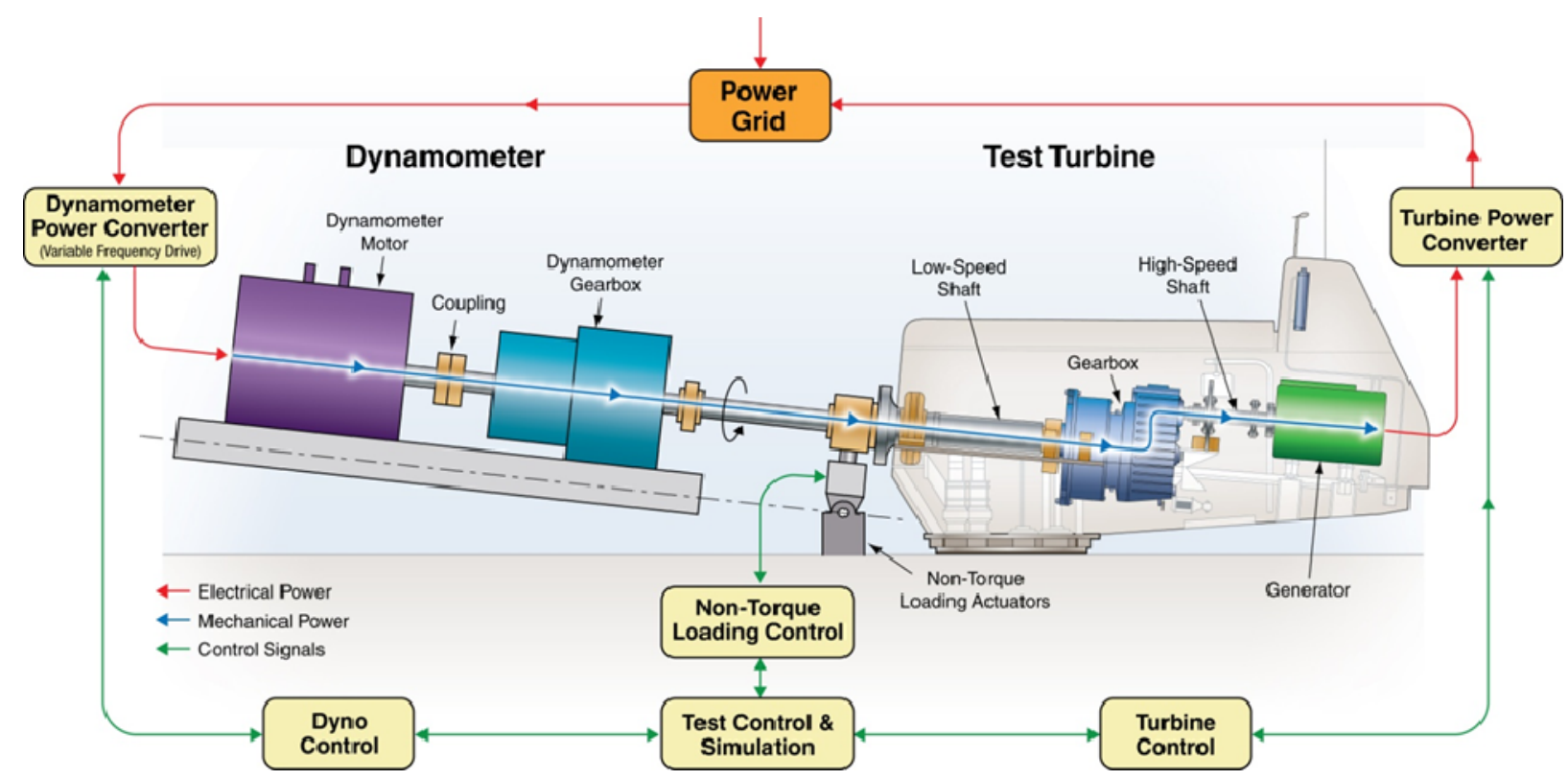

Figure 1(a). Diagram of NREL 2.5-MW dynamometer test facility

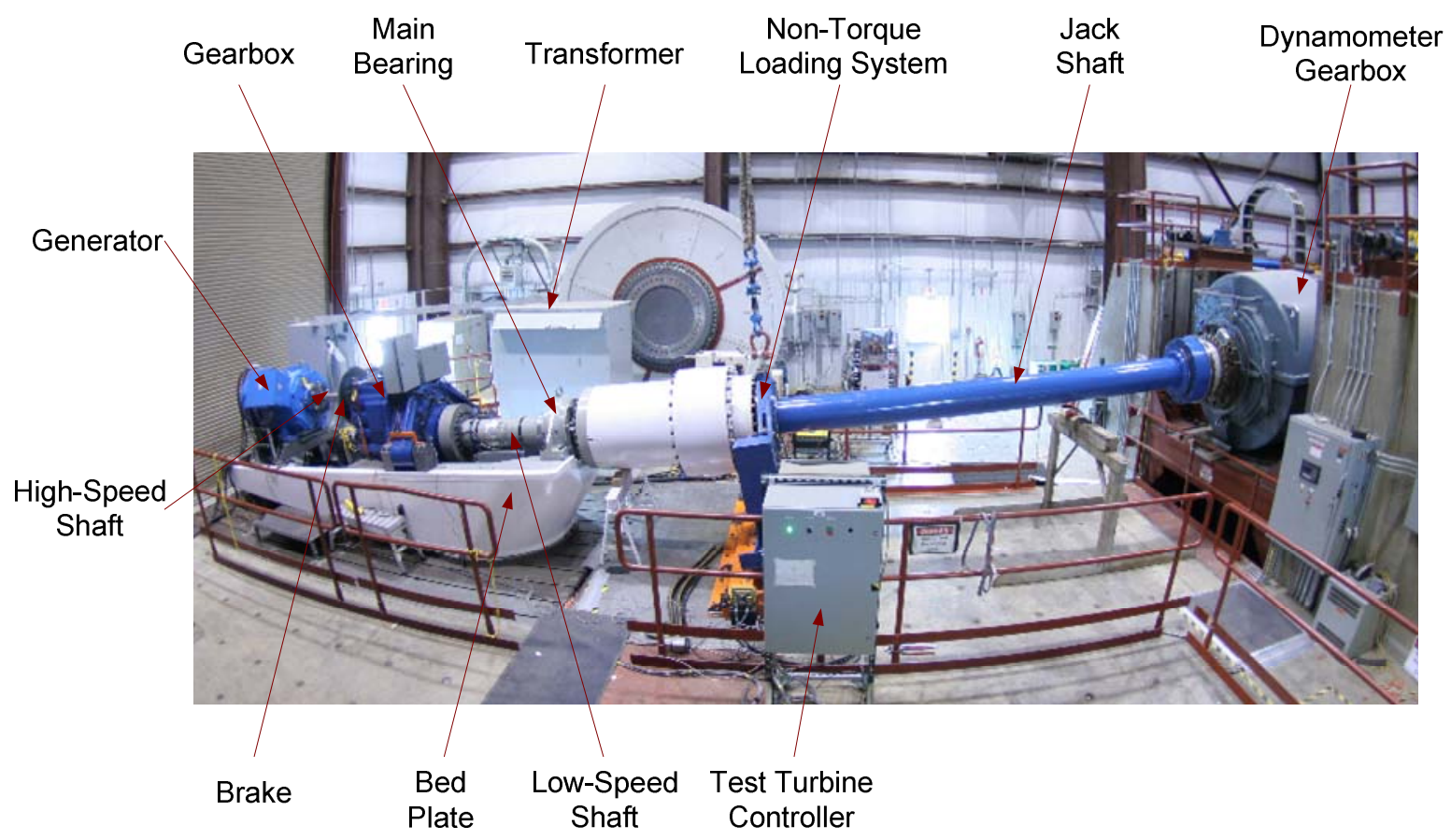

Figure 1(b). NREL dynamometer test stand with the test article installed

Figure 2 illustrates the internal configuration of the wind turbine gearbox that was tested. The analysis provided herein focuses on the high-speed shaft (HSS) and intermediate-speed shaft (ISS) gear sets located in the gearbox of the wind turbine, between the rotor hub and the generator. The damage observed on the high-speed shaft gear set was severe scuffing. The intermediate-speed shaft gear set also had scuffing and fretting corrosion on the teeth. Photos of the damage to the HSS and ISS gear set teeth are provided in Figure 3 and Figure 4. 


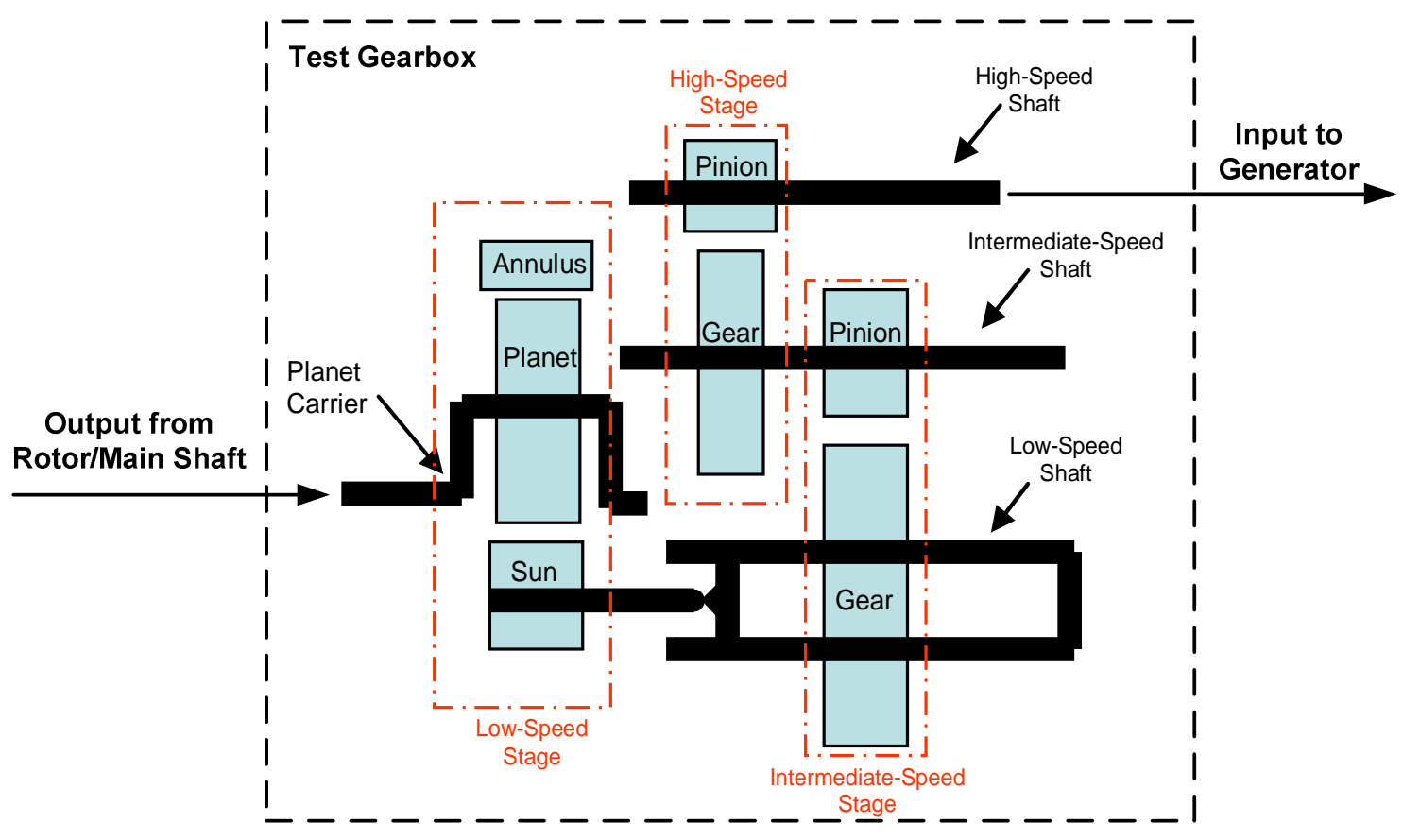

Figure 2. Test gearbox internal configuration

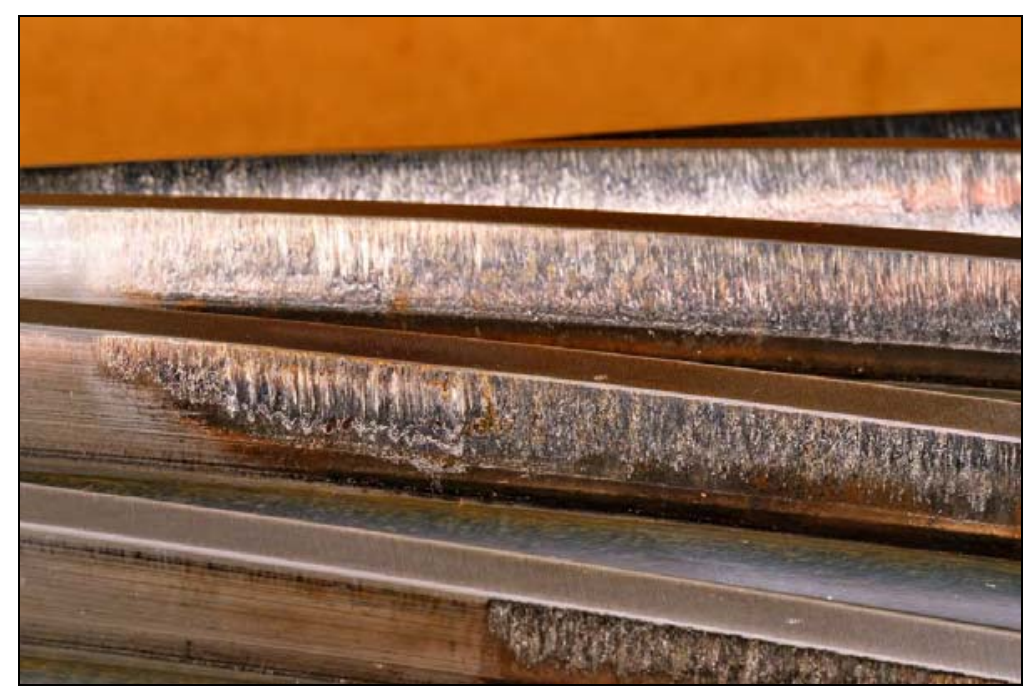

Figure 3. High-speed shaft gear set damage 


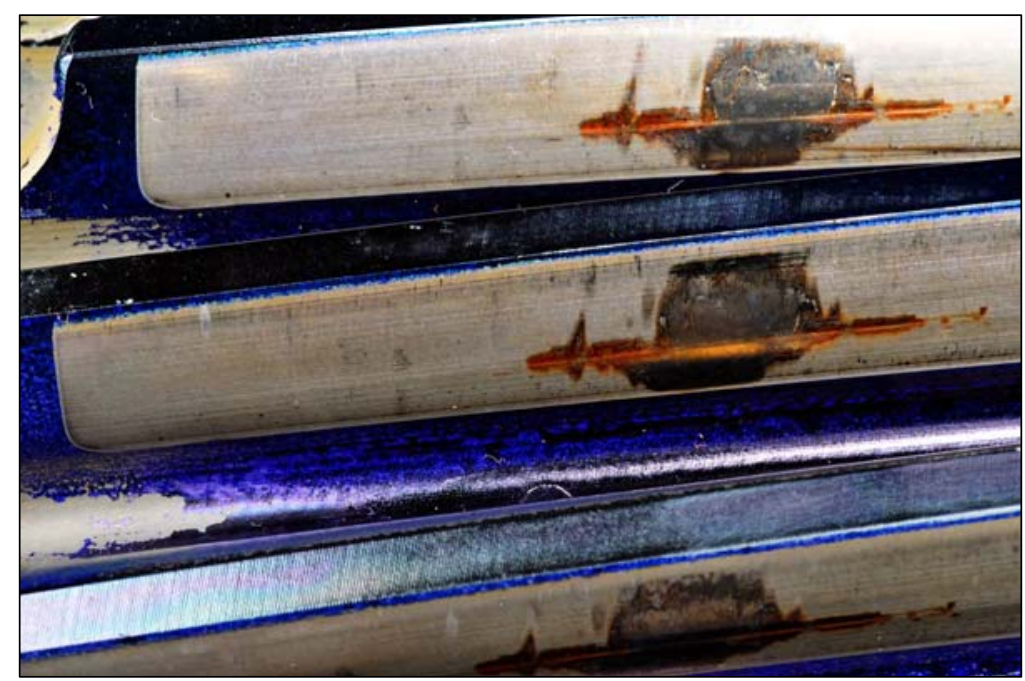

Figure 4. Intermediate-speed shaft gear set damage

The tests of the gearboxes in the wind turbine drivetrain test stand were run at three operating conditions as listed in Table 1. These tests were performed on both the "healthy" gearbox and the damaged gearbox.

Table 1. Gearbox Test Conditions

\begin{tabular}{|c|c|c|}
\hline Test Case & $\begin{array}{c}\text { Nominal HSS } \\
\text { Speed (rpm) }\end{array}$ & $\begin{array}{c}\text { Input Shaft Torque } \\
\text { (\% of rated torque) }\end{array}$ \\
\hline $2 \mathrm{a}$ & 1,200 & $25 \%$ \\
\hline $2 \mathrm{~b}$ & 1,800 & $25 \%$ \\
\hline $2 \mathrm{c}$ & 1,800 & $50 \%$ \\
\hline
\end{tabular}

For the "healthy" gearbox, the tests were conducted at the 1,200-rpm, 25\% torque and 1,800rpm, $50 \%$ torque test conditions, and each test was run for approximately 75 minutes. For the damaged gearbox, the tests were conducted using all three test conditions, and each test was run for about 15 minutes. Vibration data was measured by accelerometers placed at various locations along the drivetrain. The locations of two sensors used in this study-AN6 and AN7—are shown in Figure 5. Sensor AN6 mainly monitors the radial vibration of gears and bearings on the ISS. Sensor AN7 mainly monitors the radial vibration of gears and bearings on the HSS. Both of these sensors are integrated-circuit piezoelectric-type accelerometers and both have a sensitivity of 100 milli-Volts per g. The accelerometer data was collected at a sampling rate of $5 \mathrm{kHz}$ for 1.6 seconds every 1.5 minutes. 


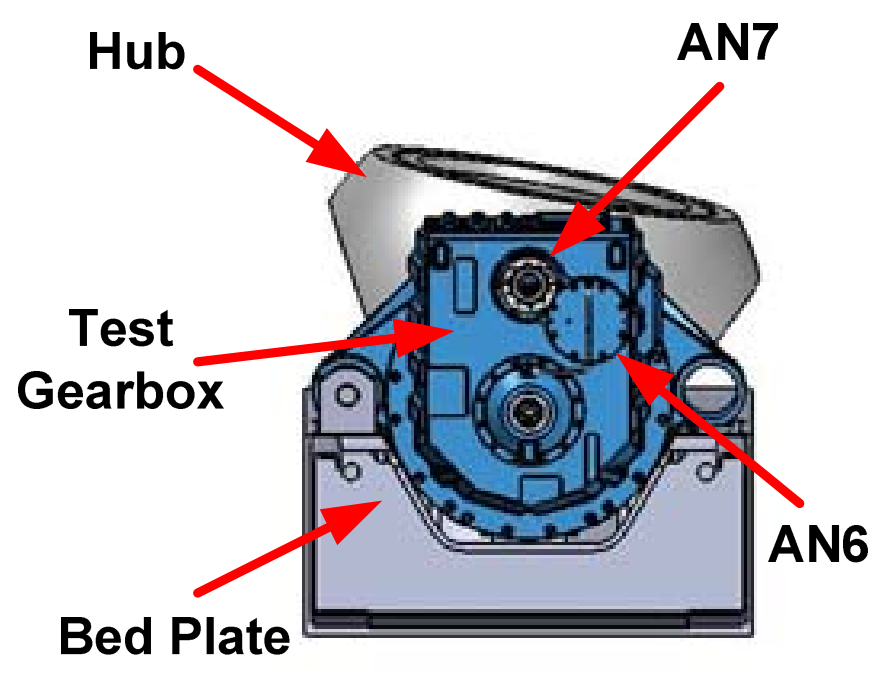

Figure 5. Location of accelerometers AN6 and AN7

Oil debris generated was measured by an inductance-type oil debris sensor installed in the main gearbox lubrication supply line. The oil debris sensor has a bore diameter of $38 \mathrm{~mm}$. It measures the change in a magnetic field that is caused by passage of a metal particle larger than 350 microns, and counts the number of particles. Filters located downstream of the oil debris sensor capture the debris after it is measured by the sensor. The oil debris sensor was engaged for the duration of each test case, and the particle counts increased whenever particles were detected by the sensor.

Additionally, torque was measured by four strain gages arranged as a full Wheatstone bridge and mounted on the input shaft to the test gearbox. The high-speed shaft rotations per minute was measured by an encoder-generating 500 pulses per revolution-mounted on the non-drive end of the generator, and which was connected to the back of the test gearbox. The torque and HSS rotations per minute were sampled at $100 \mathrm{~Hz}$ continuously on the "healthy" gearbox and at $40 \mathrm{kHz}$ continuously on the damaged gearbox. Two different sampling rates were used because for the "healthy" gearbox a data-acquisition system was developed for collecting the test gearbox dynamics data, and the configuration used a continuous sampling rate of $100 \mathrm{~Hz}$. For the damaged gearbox, a dedicated condition monitoring (CM) data-acquisition system was used, and the configuration was capable of sampling at $40 \mathrm{kHz}$.

\section{VIBRATION ANALYSIS}

Numerous diagnostic techniques have been developed from vibration data to detect damage and abnormal conditions of dynamic mechanical components. The rationale is that damaged components produce specific fault patterns in the accelerometer vibration signatures. Condition indicator (CI) refers to the vibration characteristics extracted from these signatures, and it is used to reflect the health of the component. Gear CIs are generated from fault patterns produced in vibration signatures when damaged components interact with their environments. The CI used is selected based on its ability to detect the specific component fault under investigation while 
minimizing false alarms. This requires determining both "healthy" and faulted signatures of the component under investigation, and also that a threshold be defined for the CI.

Gears produce vibration signals synchronous with shaft speed, therefore gear CIs typically are calculated from time synchronous averaged (TSA) data. "Time synchronous averaged" refers to techniques for extracting periodic waveforms from additive noise in the time domain by averaging signals over several revolutions of the shaft, thus reducing the noise in the signal. Employing TSA requires using a tachometer or a sensor that generates a pulse per shaft revolution to average the vibration data per revolution of the shaft. This type of processing based on the encoder output was not performed during testing. Additionally, during tests conducted in the test stand minor variances were observed in the speed data. For this reason, a gear CI was selected that could be calculated from spectrum (frequency) data without necessitating time synchronous averaging. Note that, in the field, the rotor speed varies based on wind conditions. This, in turn, causes varying loads and speeds on the gearbox. Depending on the variances in speed, sample rates, and sample durations used by the wind turbine condition monitoring system, a new TSA technique might need to be developed for this application.

The CI selected to detect damage on HSS and ISS gear sets is referred to as sideband index (SI) [7]. An SI is a measure of local gear faults and is defined as the average amplitude of the first order sidebands of the fundamental gear meshing frequency. An increase in the magnitude of the sidebands of the fundamental gear meshing frequency (number of teeth $\times$ shaft speed) indicates a local fault. The sidebands are the two frequencies calculated as the total number of gear teeth minus 1 multiplied by the gear rotations per minute and the total number of gear teeth plus 1multiplied by the gear rotations per minute. Table 2 provides details of the two gear sets and frequencies based on shaft speeds. Due to the minor speed variances during testing and the lack of a signal pulse per shaft revolution, the frequency range used to calculate the sidebands was increased to include the speed variation. The maximum amplitude within the two sideband frequency ranges then was determined. The average amplitude of the two sidebands within this range then was equal to the SI.

Table 2. Gear Dimensions

\begin{tabular}{|l|c|c|c|}
\hline Gear Element & Number of Teeth & Speed (rpm) & $\begin{array}{c}\text { Gear Meshing } \\
\text { Frequency (Hz) }\end{array}$ \\
\hline ISS gear & 82 & $84 / 126$ & \multirow{2}{*}{$115 / 173$} \\
\hline ISS pinion & 23 & $300 / 450$ & \\
\hline HSS gear & 88 & $300 / 450$ & \multirow{2}{*}{$440 / 660$} \\
\hline HSS pinion & 22 & $1,200 / 1,800$ & \\
\hline
\end{tabular}


The spectrum data used to calculate SI for the HSS and the ISS gear sets was collected at a sampling rate of $5 \mathrm{kHz}$, for 1.6 seconds every 1.5 minutes. Only 15 spectra were analyzed for the conditions 2a and 2c for the "healthy" gearbox, and 8 spectra for the 2a to 2c damage conditions from the two accelerometers (AN6, AN7). Spectrum data from the $2 b$ "healthy" gearbox condition was unavailable, therefore only SI obtained from 2a and 2c is discussed. For illustrative purposes, all of the spectra for this analysis from the two accelerometers were averaged and plotted in Figure 6 and Figure 7. Although scales can be expanded to compare the change in amplitudes for different conditions, visually analyzing this data is a time-consuming process. Condition indicators enable reducing each spectrum down to one parameter for each acquisition. Damage progression could be determined by monitoring the CI along with torque, speed, and debris generated.

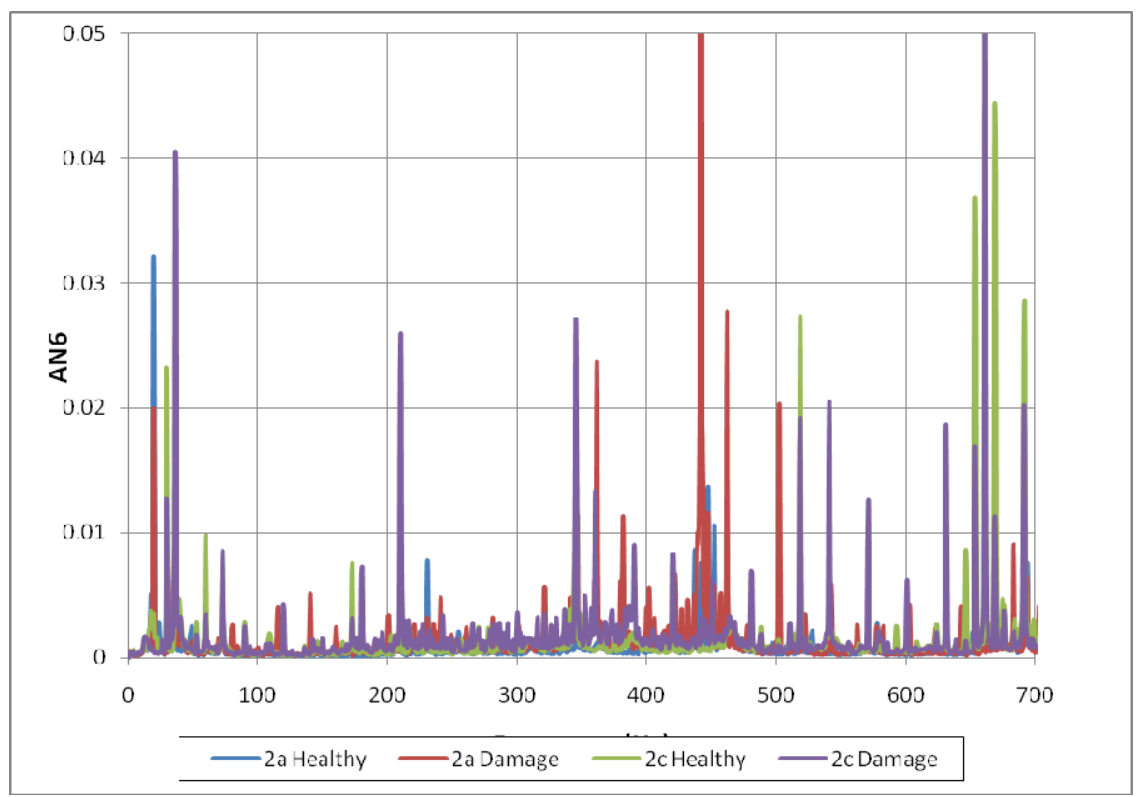

Figure 6. AN6 average spectra data 


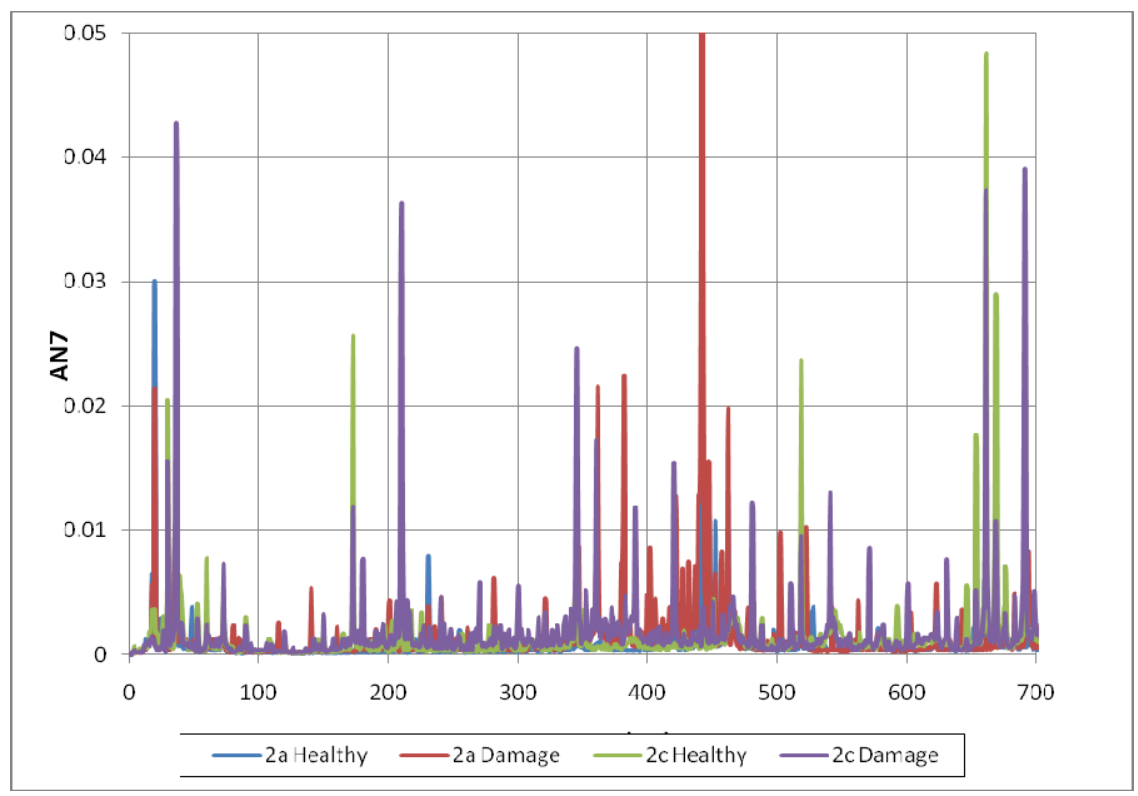

Figure 7. AN7 average spectra data

Figure 8 and Figure 9 are plots of SI for the gear sets at the 2a and 2c conditions for the "healthy" and damaged gearboxes. The readings represent an elapsed time of about 70 minutes for the "healthy" gearbox and 10 minutes for the damaged gearbox during each acquisition. Figure 8 is a plot of the SI for the HSS gear set calculated from vibration data measured with accelerometer AN7. Based on this small data set, a threshold could be set at 0.0125 to indicate damage to this gear set for both test conditions. The values were greatest for $2 \mathrm{c}$, the increased speed and load conditions.

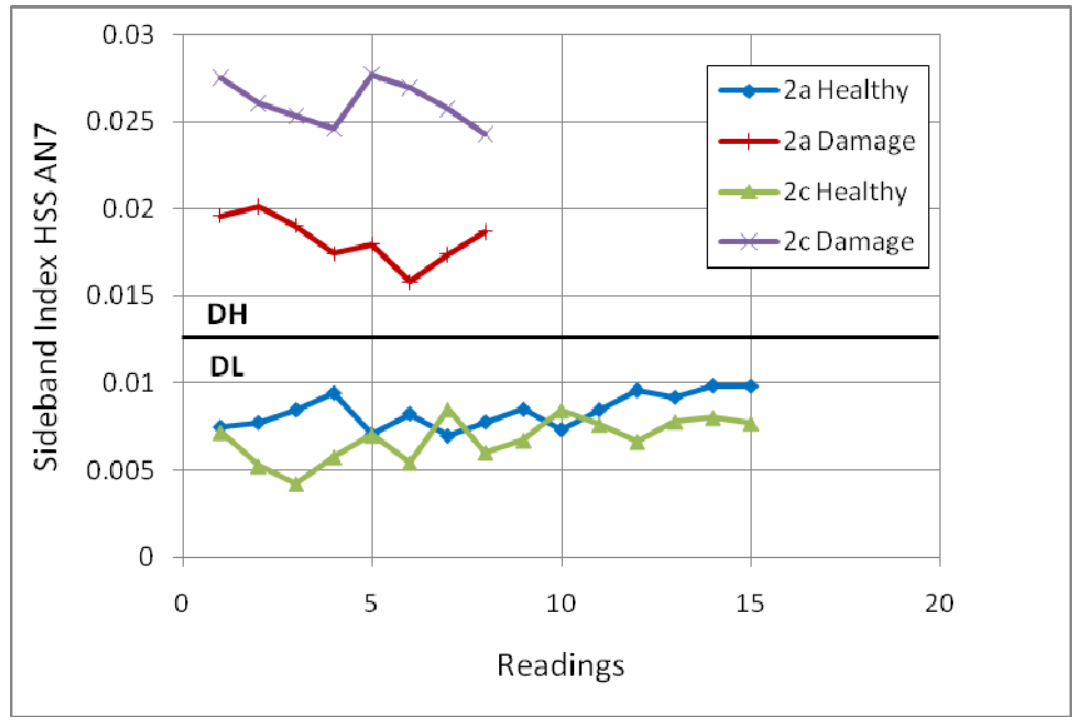

Figure 8. Sideband index for high-speed shaft gear set from AN7 
Figure 9 is a plot of the SI for the ISS gear set calculated from vibration data measured with accelerometer AN6. Based on this small data set, a limit could be set at 0.003 to indicate damage to this gear set at the greater load and speed conditions. At the lesser load conditions, it was more difficult to differentiate between the damaged and "healthy" gears. The magnitude of the sidebands measured with this accelerometer, AN6, at sidebands for the ISS gear mesh frequencies also were significantly less than those measured for the HSS gear with AN6. The SI values for the ISS gear using the AN7 accelerometer also were comparable to AN6 SI values. This indicates that many factors affect the response of a sensor to a specific fault. Factors that affect vibration response can include accelerometer type, location, mounting, and signal processing. Gear speed, applied torque, and system structural dynamics also can have an effect. The relationship between all of these variables is complex and not well defined. Determining factors with the greatest effect (variability) on the CI response requires further study.

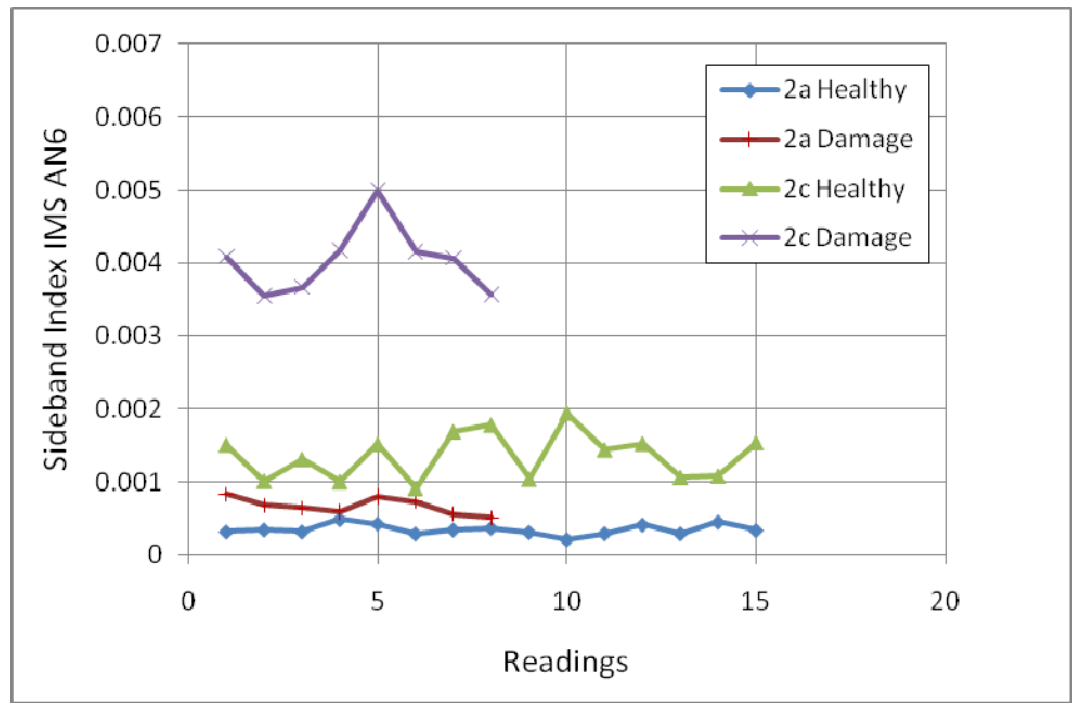

Figure 9. Sideband index for intermediate-speed shaft gear set from AN6

\section{OIL DEBRIS ANALYSIS}

The baseline oil debris data was collected from the gearbox after its run-in was completed and under "healthy" conditions. The oil debris sensor was located in the main lubrication loop of the test gearbox. It monitored lubrication oil for ferrous particles larger than 350 microns in size. Downstream of the sensor, the oil system had a 50-micron and 10-micron 2-stage filtration system to prevent debris from being recirculated within the lubrication system. The oil system also had a 3-micron offline filtration system that could run continuously 24 hours a day, 7 days a week, even when the gearbox is not being tested. The "healthy" gearbox was run for 4 hours; during that period, a maximum of 11 particles were detected by the oil debris sensor. The 11 particles detected were used as the baseline particle count.

When the damaged gearbox was tested at the three conditions, debris was detected by the oil debris sensor. A plot of the change in debris during testing of the three conditions is shown in Figure 10. The assumption that the debris was generated only by the damaged ISS and HSS gears 
during testing could not be verified. The scanning electron microscope (SEM) analysis of the filter cloth used to drain the gear oil during the test gearbox disassembly indicated that the major particulate constituents in the specimen were steel, iron oxide, brass, and zinc. Additional damage propagation tests are required to correlate the tooth damage to oil debris generated. For the purposes of this analysis, it was assumed that the debris measured by the oil debris sensor came from the gears during testing. The period in which this data was collected for the three conditions also is listed in the Figure 10 legend. They are longer than the vibration datacollection periods for the damaged gearbox tests, as shown in Figures 8 and 9. The reason for the difference in collection periods is that each test involved start-up and coast-down processes, during which the oil debris sensor was functioning, and the vibration spectra evaluated only were extracted when the test gearbox settled into stable operational states. The readings are comparable to minutes. For demonstration purposes, if the gear-damage level and progression could be verified during the period when oil debris data was collected, then a threshold for the oil debris sensor could be set based on the number of particles detected.

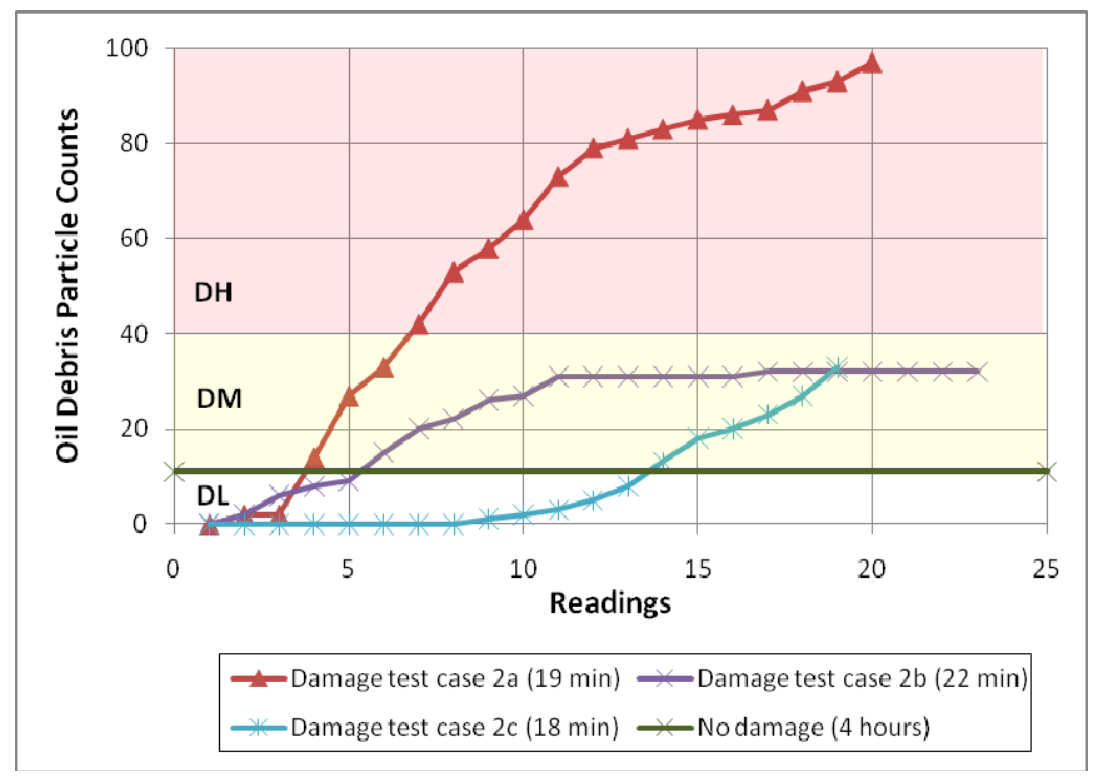

Figure 10. Oil debris particle counts

\section{DATA FUSION}

Prior to review of the test results, data fusion analysis techniques were to be applied to the vibration and oil-debris data, mapping the oil debris generated and the vibration condition indicator to gear damage. The rationale is to combine the capability of pinpointing damage location by the vibration CI, with the damage severity estimated by the oil debris analysis. Multisensor data fusion works in much the same way as the human brain; it integrates data from multiple sources and uses it to make decisions. One objective of condition monitoring is to make it easier to make a decision about—or take action on—-the current state of the system. Data fusion is one method that combines historical knowledge of the system to improve a maintenance person's ability to make decisions regarding the health of the system. Decision-level fusion was 
used to integrate these inputs because it does not limit the fusion process to a specific feature, thus enabling different features to be used without changing the entire analysis. This requires assessing the performance of the individual measurement technologies prior to their integration. If the individual condition indicators have low detection rates and high false alarm rates, then little is gained by integrating the technologies.

An understanding of the strengths, weaknesses, and constraints of each measurement technology is required before the strengths can be capitalized upon via data fusion. A review of the data at the two damage conditions indicates that the vibration CI detected the damage to the HSS gear set at both load and speed conditions, and detected damage to the ISS gear set at the high load and speed conditions. The poor performance of the CI for this gear set could be due to many environmental factors; this warrants further investigation. Other gear CIs also could be investigated. The small amount of data collected by the oil debris sensor made it difficult to obtain inferences on its performance. Further analysis of the individual CIs is required before a fusion model can be developed. For demonstration purposes, a data fusion model for this application is briefly discussed. Decision-level fusion is a method that can be used to integrate multiple inputs. Fuzzy inference can be used to fuse the information. A detailed description of the process used to define the membership functions can be found in Dempsey [8]. Input values are defined, such as oil debris particle counts, vibration CI sideband index, component (HSS gear), torque, and speed with thresholds that correlate to damage levels. Using rules and membership functions, outputs per damage levels can be defined such as damage low (DL), damage medium (DM), and damage high (DH). These damage levels can be correlated to specific maintenance actions such as no action, inspect, and repair. After more data becomes available, a data fusion model such as that shown in Figure 11 can be developed for this application. 


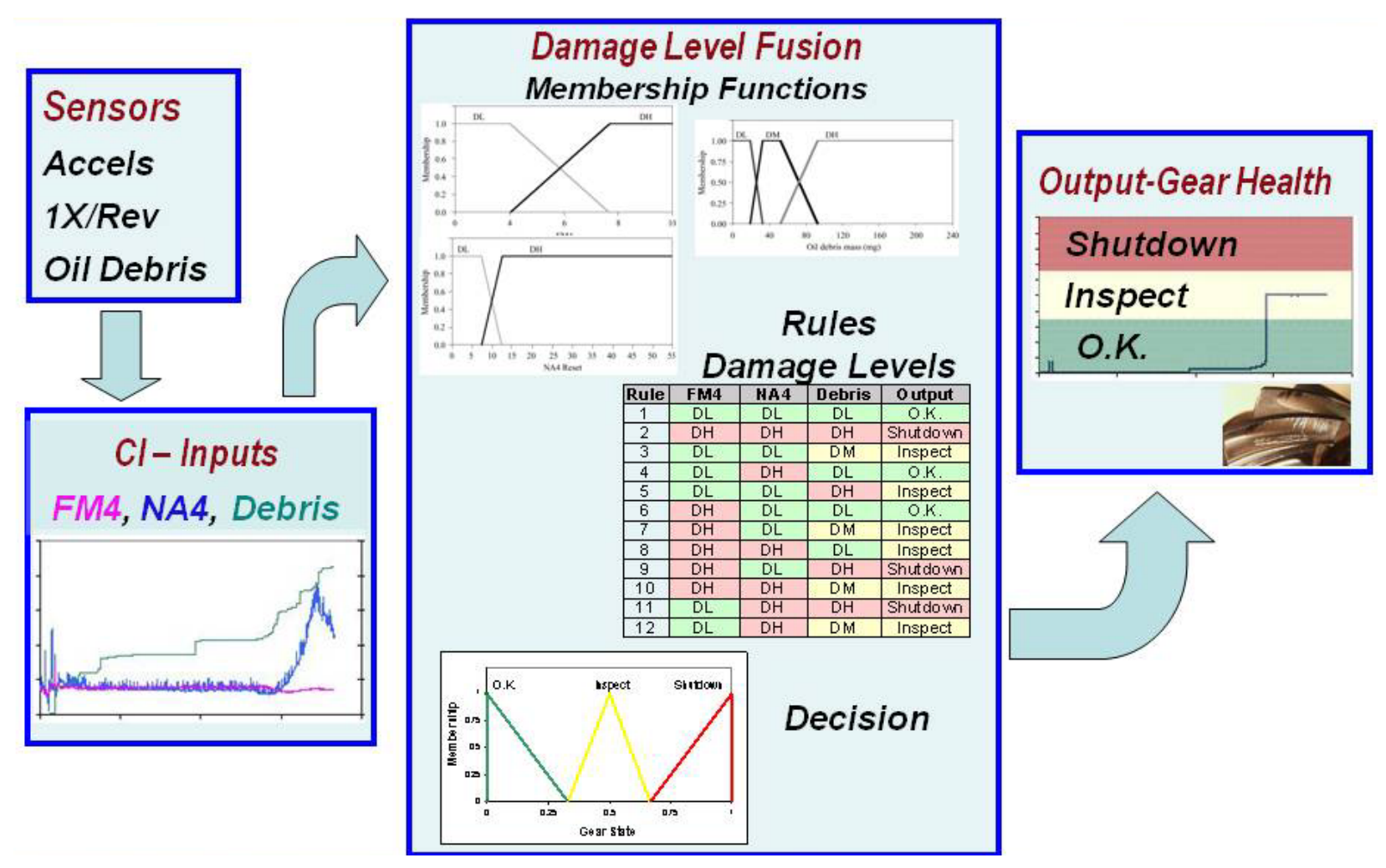

Figure 11. Fusion applied to rotorcraft spiral bevel gears

\section{CONCLUSIONS}

The objective of this research was to demonstrate that, when combined, vibration-based healthmonitoring techniques, oil debris analysis techniques, and wind turbine operational parameters provide improved detection and decision-making capabilities as compared to that provided by individual diagnostic tools. Data was collected from a "healthy" gearbox and a damaged gearbox - both tested on the NREL dynamometer test stand. Vibration and oil debris data were collected along with torque and speed data. Results indicate that the vibration-based condition indicator sideband index can be used to indicate damage to gear teeth of the HSS gear set. Damage to the ISS gear set only could be detected at the greater speed and load test conditions. Oil debris particle counts also increased when the gearbox with damaged components was tested. Further research is required to improve the performance of the individual health-monitoring tools. This can be accomplished by defining thresholds for individual measurement technologies. This will enable detection rates and false alarm rates to be quantified for individual measurement technologies. Quantifying the strengths, weaknesses, and constraints of each measurement technology currently used to monitor wind turbine health—and then capitalizing on these strengths via data fusion—is key to the development of future health-monitoring systems. 


\section{ACKNOWLEDGEMENTS}

The authors thank the U.S. Department of Energy for its support of this work. We also acknowledge and appreciate the support given by the NREL condition-monitoring partners.

\section{REFERENCES}

1. Caselitz P, Giebhardt J. Rotor condition monitoring for improved operational safety of offshore wind energy converters. Journal of Solar Energy Engineering 2005; 127(2): 253-261.

2. Gonzalez JR, Velayos J, Comamala M. Predictive maintenance of cogeneration engines through the analysis of the circulating fluids. ASME Conference Proceedings 2002; (36177) 389396.

3. Dempsey PJ, Handschuh RF, Afjeh AA. Decision fusion analysis detects spiral bevel gear damage. IEEE Aerospace and Electronic Systems Magazine 2003; 18(9): 11-17.

4. Sheng S, Gao RX. A wavelet-based fuzzy data fusion scheme for bearing defect severity classification. ASME Conference Proceedings 2003; (37130): 1285-1292.

5. Musial W, McNiff B. Wind turbine testing in the NREL dynamometer test bed. WindPower 2000 Conference, April 30-May 4, 2000; Palm Springs, CA.

6. National Renewable Energy Laboratory. 2.5 MW Dynamometer Testing Facility Fact Sheet. [Online]. Available: http://www.nrel.gov/docs/fy11osti/45649.pdf. (Accessed May 5, 2011.)

7. Antolick LJ, Branning JS, Wade DR, Dempsey PJ. Evaluation of gear condition indicator performance on rotorcraft fleet. Proceedings of the American Helicopter Society 66th Annual Forum, May 11-13, 2010; Phoenix, AZ.

8. Dempsey PJ. Integrating oil debris and vibration measurements for intelligent machine health monitoring. PhD Thesis, The University of Toledo, 2002. 